\title{
PROMOTORAS COMUNALES... DESCOMUNALES!!!
}

\section{Ordinary community promoters... Estraordinary ones!!!}

Martha Llanos Zuloaga*

\begin{abstract}
Resumen
La autora relata sus vivencias personales en los Andes y el Himalaya como un tributo a las mujeres promotoras comunitarias de dos Continentes América del Sur y de Asia del Sur. Los perfiles que surgen de estas Animadoras, Promotoras expresan una serie de valores especiales en su desarrollo personal como colectivo y que a través del propio trabajo se incrementa y proyecta al servicio de los demás, la formación que reciben se centra en procesos de enseñanza aprendizaje en donde vemos que la mayoría de programas en ambos países tiene una orientación en la acciónreflexión -acción base de una línea de pensamiento Freiriano en total vigencia y expansión actual.
\end{abstract}

Palabras clave: Promotora comunal

\begin{abstract}
The author shares her personal experiences in the Andes and the Himalayas as a tribute to the women community promoters of two continents, South America and South Asia. Profiles emerging from these Animators-Promoters express a number of special values in their personal and collective development, and that through their own work service such development increases and benefits others. Their training focuses on teaching and learning processes where we see that most of the programs in both areas are oriented to the action-reflection-action based on a line of Freirian thought in full force and current expansion.
\end{abstract}

Key words: Community promoter.

* Profesora Investigadora Extraordinaria, Universidad Femenina del Sagrado Corazón. Directora Relaciones Internacionales Foro Red Paulo Freire 
Es preciso potenciar el valor de las niñas para sus propias familias y para la sociedad y reforzar esa imagen con la adopción y aplicación de políticas educacionales y sociales que fomenten su plena participación en el desarrollo de la sociedad en que viven. CONFERENCIA INTERNACIONAL DE POBLACION Y DESARROLLO,1994.

Vivimos en un mundo de símbolos y metáforas, la cosmovisión de los pueblos originarios nos habla de la madre tierra, más conocida en el mundo Andino como la Pacha Mama, es esta Pacha Mama la que representa el símbolo de lo femenino con sus características de tierras fértiles, acogedoras, proveedoras de alimento, de protección, de siembra y cosecha para la nutrición y es precisamente ella la que está presente en esta celebración del llamado día de la Mujer.

Desearía que este relato de vivencia personal sea un tributo a las mujeres promotoras comunitarias de dos Continentes en los que transcurrieron y transcurren experiencias inéditas de mi peregrinaje, el de compartir la vida en lugares caracterizados por dos cadenas de montañas que simbolizan la fortaleza, seguridad y majestuosidad y poder: los Andes y el Himalaya, en dos Continentes: América del Sur y de Asia del Sur y específicamente recorrer dos culturas milenarias, cuyas historias comparten situaciones similares por procesos histórico culturales parecidos. Ellos son Perú y Nepal.

Estos países son el escenario para este tributo de aprecio y diseminación de las acciones de mujeres rurales que sufren las marginaciones propias de sistemas patriarcales, de incumplimiento de sus derechos humanos a la educación y salud, mínimo respeto a sus culturas y un agobiante sistema de marginación social por factores culturales o de jerarquía de castas.

Quiero resaltar y testimoniar la presencia en ambos países de las Promotoras Comunitarias, que recibiendo nombres diversos las podemos identificar como Animadoras, Facilitadoras, promotoras etc. y que actúan específicamente en campos de desarrollo social vinculados a la educación, salud y desarrollo agrícola entre otros. Todas ellas proceden de las mismas comunidades en donde laboran en condiciones muchas veces de voluntariado o con mínima remuneración, pero que sin embargo constituyen un ejemplo extraordina- rio de liderazgo femenino que tiene un impacto muy grande tanto en sus vidas (personal, familiar, profesional y comunitaria) como en el aporte al cumplimiento de los derechos del niño, de un desarrollo sostenible, de la protección ambiental y de la salud de las poblaciones.

\section{PERÚ. MUJERES INOLVIDABLES CONSTRUC- TORAS DE UN MUNDO MEJOR}

La experiencia personal como trabajadora en desarrollo humano y comunitario me ha llevado a lo largo de cuatro décadas a compartir con Promotoras en diversos programas en el territorio peruano y en otras partes del mundo. En este acápite me referiré más específicamente de las llamadas Animadoras de los PRONOEI o programas no escolarizados de Educación Inicial. El perfil de Animadora o Promotora, tiene como componente básico el valorizar su condición de voluntaria no profesional de la comunidad, su rol de madre, de experiencia con los niños y o adultos, su compromiso en la búsqueda de alternativas educativas de su comunidad, su membrecía comunal y el ser propuesta por la comunidad, que interviene en el proceso de su selección, y su nivel de instrucción no es un factor excluyente.

En la formación la línea de acción- reflexión-acción está presente en la relación de aprendizaje horizontal, de educador - educando entre la docente - coordinadora (profesional) y la animadora (voluntaria) que permite la interiorización de principios y pautas de trabajo y por ende garantiza la transferencia del aprendizaje a situaciones concretas con los niños. Asimismo en la constitución de grupos de aprendizaje que favorece el ambiente propicio para vivir, compartir y reflexionar sobre las experiencias y situaciones acontecidas.

Es relevante indicar el contexto familiar y comunitario, local y regional son tomados en cuenta como factores de enseñanza, y aprecian el valor de las no profesionales o promotoras comunitarios (prioritariamente son mujeres) especialmente al grueso de mujeres gestoras promotoras que actúan como un puente entre los educadores profesionales y la comunidad. Esta inclusión de Promotoras Comunitarias ha generado en el caso de Perú un espacio de oportunidad para el descubrimiento de la capacidad de gestión de las mujeres, 
de sus estilos armónicos de liderazgo y de su gran resiliencia haciendo viable y con múltiples ventajas su inserción como Promotoras de la Comunidad y su participación en cualquier acción educativa.

Las Promotoras Comunales y o Animadoras constituyen un valiosísima grupo humano que en forma bastante silenciosa son fundamentales en el Desarrollo Comunitario. La gran mayoría son mujeres seleccionadas en la misma comunidad; generalmente pertenecen a la misma clase social y cultura que los niños/ niñas/adultos beneficiarios de los proyectos y que son escogidas en base a su compromiso con la tarea de desarrollo, educativa en campos de salud, agricultura, planeamiento y otros. Ellas contribuyen al crecimiento de las potencialidades locales y a la creación de un genuino sistema de reciprocidad, en el que se benefician tanto las familias, los promotores, como la comunidad en su totalidad. A través de esta estrategia los programas han tenido una inserción más adecuada en sus localidades de trabajo; han respetado los liderazgos existentes y han propiciado nuevos canales de participación fortaleciendo los recursos humanos locales y el empoderamiento de las mujeres como gestoras de desarrollo.

"Ustedes saben que desde los quince anos trabajé como animadora de educación inicial, luego alfabetizando..."

"Mi práctica es de construir. Desde los doce años ayude a mi madre a levantar locales comunales, postas medicas, programas no escolarizados, PRONOEIS, plantar árboles, ...”.

María Elena Moyano: En busca de una esperanza

La vida de María Elena Moyano, nos ha demostrado su gran poder de Resiliencia, que ante adversidades de la vida es posible ver una luz a través del reconocimiento de nuestras capacidades como mujeres, capaces de transformar el contexto inmediato y mas allá el mundo si nos lo proponemos, pienso que la referencia de las vidas de las animadoras tienen un gran valor si las vemos como mujeres que se reconocen como sujetos autónomos y con la capacidad de realizar sus metas a través del desarrollo de su trabajo en su hogar y en su comunidad. Algunos testimonios de Promotoras nos indican:

"Elevé mi autoestima, yo estaba pasando por momentos tristes en mi hogar me había separado de mi esposo, estaba sola con mis hijos, he sufrido. Fue muy bonito sentir que allí iba a empezar a ganar algo, ahí una propina que recibía con mucho agrado para mis niños y a parte quería servir a la comunidad, poder servir, lograr que esos niños tengan una vida diferente" Nelly, Andahuaylas.

“... las madres, así como yo son tímidas tienen recelo a las personas, si alguna vez tenemos alguna oportunidad, donde hayan personas que nos quieran ayudar. Porque uno pone un poco de valor, y sé qué vamos a ir adelante muy bien. Nunca debemos quedarnos atrás, porque quedarnos atrás es como estancarnos. Entonces yo les pido a las madres que participen en nuestros PRONOEI." Victoria, Cuzco.

"Vengan, que nosotros en ningún momento les vamos a cerrar las puertas, aprendan y sobresalgan así como yo, para que más tarde den gracias y tengan esa hermosa experiencia, la que yo he vivido durante esos cinco años. Estoy muy contenta y espero que todas ustedes sigan así adelante”. Olga, Vitarte.

En la actualidad y habiendo asumido la Dirección de la línea de Arte y Educación Popular el Foro Paulo Freire del Perú está dando inicio a la utilización del Arte y es así que a través de Teatro del oprimido se fortalecerá la formación, intercambio y análisis crítico entre aquellas Promotoras gestoras de desarrollo personal y colectivo, con encuentros en diálogos intergeneracionales entre Ex Animadoras-Promotoras y las nuevas Promotoras Comunales.

\section{NEPAL, LA GRANDEZA HUMANA TIENE ROS- TRO DE MUJER}

Viví en Nepal e India en la década de los años 90, es en Nepal en donde la similitud geográfica con el Perú especialmente nuestras zonas andinas es extraordinaria, las personas me hicieron siempre recordar a nuestra zona andina, Cusco, Puno, Ayacucho, Apurímac lugares en donde he trabajado especialmente con comunidades indígenas. Regresé el 2008 y el 2009 a Nepal, habían transcurrido 12 años, en estos 12 años Nepal sufrió la problemática de conflicto armado interno, con la presencia de un movimiento maoísta, historias que reflejan nuestra similitud a pesar que estamos en continentes diferentes. 
La situación de la mujer en Nepal es bastante difícil, la marginación es alta y sus niveles educativos limitados con aproximadamente solo un 30\% de mujeres alfabetas. Recuerdo como le apostamos en la década del 90 en que trabajé en la UNICEF Nepal como Responsable de Educación, a la formación de las profesoras mujeres, a las Promotoras Comunales mujeres y basada en la experiencia peruana me permitió colaborar con enfoques que fueran de formación personal y comunitaria. Nuestro lema en aquel entonces fue "Andes e Himalaya unidos por los niños/as de Nepal." La base fue que las montañas son fuerza y que las mujeres representaban representaban las fuerzas de esas montañas. La ayuda de cooperación técnica también vino de colegas de América Latina (Rosa María Torres entre otras).

Recientemente al volver a Nepal encontré unas historias de éxito, de liderazgo de las mujeres, de amor incondicional que a pesar de haber tenido un periodo sangriento y cruel en donde como en cualquier situación de emergencia armada las víctimas son siempre las niñas, niños y las mujeres. Sin embargo las Mujeres de Nepal nos han dado ejemplos de su valentía y coraje y de la unión para afrontar problemas y marginaciones culturales y de género.

Ellas crearon lo que se llama las Mujeres del Everest y en el 2009, diez mujeres nepalesas (periodistas, promotoras, profesoras, amas de casa, modelo, lavandera y otras) participaron en la primera expedición al Everest totalmente integrada por mujeres, que escaló con éxito el pico más alto (29.000 pies, es decir más de 8.800 metros). Debieron superar todos los obstáculos que suelen enfrentar los alpinistas, incluidos avalanchas, temperaturas extremadamente bajas, tormentas repentinas y bajos niveles de oxígeno, ellas los superaron todos.

Ahora, siete de ellas han emprendido otra misión: visitan escuelas para hacer presentaciones en vídeo sobre su expedición. El objetivo es educar y servir de inspiración a las niñas y niños en relación con temas prioritarios como la igualdad entre los géneros, el empoderamiento de la mujer y el calentamiento del planeta.

Durante las visitas a las escuelas el grupo habla de sus experiencias personales. Colaboran con las traba- jadoras Comunitarias con las Maestras y madres de familia. Las mujeres provienen de distintos estratos sociales y castas, pero trabajaron juntas como un equipo. Entre sus testimonios nos indican:

"Queremos demostrar a los niños que el éxito se obtiene mediante el trabajo arduo y la educación; que ni la edad, ni la religión, ni la casta ni la región de donde se proviene importan si realmente quiere lograrse algo", Shailee Basnett.

Las mujeres del Everest también están seriamente preocupadas por los efectos del calentamiento del planeta en el Himalaya, uno de los grandes tesoros de Nepal, y en los medios de subsistencia del pueblo nepalés. Gracias a los esfuerzos de este grupo, el Monte Everest ahora sirve para llamar la atención sobre la igualdad entre los géneros y el rol de las mujeres como gestoras del desarrollo.

\section{REFLEXIONES}

Algunos conceptos básicos nos permitirán hacer un collage de reflexiones en torno a las experiencias vividas. Según Palomino (1991) define a los Promotores/as: son quienes impulsan y llevan a cabo las acciones con las poblaciones de base y responden por las metas comprometidas en los proyectos. Ello acarrea consecuencias que los afecta como personas, en su vida cotidiana y en el desempeño y la eficacia de su trabajo.

La Comunidad según Anderson se refiere a un conjunto de individuos que comparten un territorio geográfico. Además de compartir cultura, un medio de comunicación, expresiones (interacciones, valores, esperanzas, valores, creencias y significados) comportamientos humanos que tienen un sentido y expectativas entre sus miembros. Las comunidades son la base fundamental de las futuras naciones definidas como comunidades imaginadas, limitada y soberana

Los perfiles que surgen de estas Animadoras, Promotoras expresan una serie de valores especiales en su desarrollo personal como colectivo y que a través del propio trabajo se incrementa y proyecta al servicio de los demás, la formación que reciben se centra en procesos de enseñanza aprendizaje en donde vemos que la mayoría de programas en ambos países tiene una orientación en la acción-reflexión -acción base de 
una línea de pensamiento Freiriano en total vigencia y expansión actual.

En el devenir de la historia humana lo grupal ha sido un desafío para el desarrollo de la misma y la producción de sentido así como de la cultura ciudadana, la cultura de la convivencia, la cultura del trabajo y de la recreación en las diversas sociedades. Hoy se torna fundamental ubicar la mirada y la reflexión sobre los sucesos que acontecen en los colectivos y grupos apostando a facilitar y propiciar el protagonismo creativo de cada quién, enlazado a una trama grupal.

Para ello, en la formación y coordinación de grupos es clave el contar con esquemas conceptual - operativo desde el cual se desarrollen diversas estrategias, tácticas, técnicas pertinentes y adecuadas para el mejor desarrollo personal y comunitario. Es así que una instancia valiosa de aprendizaje-formación dirigida a todas aquellas personas que trabajan lo grupal en los campos de la salud, la educación, lo organizacional y lo comunitario, implica reflexión desde la práctica, con técnicas que facilitan la conciencia crítica, la re significación y el intercambio.

La Educación Popular tiene su punto de partida en la cultura popular, la cual posee una riqueza, diversidad y características dinámicas, pero que aun así se debe entender que es una cultura dominada. Vemos que aun cuando ha habido esfuerzos para fortalecer la interculturalidad y la comprensión de las culturas, hay una cultura "oficial" estructurada y coherente con su proyecto social, y una cultura popular popular dispersa y marginada, luchando por mantener una identidad propia frente a la cultura dominante y autoritaria.

Este marco de la Educación Popular, representa el enfoque más original surgido en América Latina, y por otro lado, existe mayor sistematización de su teoría y práctica. La problemática del trabajo con promotores encuentra que los aspectos pedagógicos, didácticos y humanos de este enfoque son de mucho valor y vigencia. Es así que se rescatan saberes, propicia el dialogo, asigna valor a todos y cada uno de los y las participantes, es decir un modelo de abajo hacia arriba, basado en las necesidades de las personas locales y asegurando que las comunidades participen en la identificación y solución de sus problemas. De esta forma considera al desarrollo como el desarrollo del potencial humano y social, un proceso en el cual las personas, se organizan y actúan para resolver sus problemas.

El proceso de "acción-reflexión - acción" en el cual las personas pueden analizar y volverse concientes de sus posibilidades y del valor de las interacciones humanas, es así que el descubrimiento de sus poderes, de sus potencialidades, especialidades natas y la riqueza de la propia diversidad, repercute en su autoestima, liderazgo y se vuelven conscientes de que hay algo que pueden hacer para cambiar sus vidas y empiezan a construir las estructuras organizativas y administrativas para lograr el cambio.

El papel del promotor/a trabaja al lado de las personas, niños y adultos, estimulando el descubrimiento de las potencialidades. Su tarea fundamental es motivar la expresión abierta de alternativas, ideas, soluciones, contribuyendo a que las personas consoliden su seguridad y la identidad personal que ayudara a la construcción de herramientas grupales para el desarrollo comunitario.

Paulo Freire en "La educación como práctica de la libertad" (1964) y "Pedagogía del oprimido" (1970) así como Educación para la Autonomía y sus últimos aportes muestran una profunda y constante evolución, en la que la transformación educativa no puede darse al margen de las demás prácticas de las instituciones sociales que conforman una sociedad. Paulo Freire decía: “... Sueño con una sociedad reinventándose de abajo hacia arriba, donde todos tengan derecho a opinar y no apenas el deber de escuchar. Este es un sueño históricamente viable, pero demanda que la gente anteayer hubiese descruzado sus brazos para reinventar esa sociedad".

Otro gran maestro latinoamericano Patricio Cariola se refiere a la Educación Popular como "educación participatoria" y establece una división entre "integrativa" - que busca aumentar la participación dentro del orden social existente -, y la "libertadora", que pretende la transformación de las instituciones y estructuras existentes.

En el día de la mujer es necesario enfatizar que la principal característica que un líder puede desarrollar se encuentra en el ser, es decir, en la práctica y vivencia de valores. En el caso de las mujeres, el liderazgo 
femenino nace del reconocimiento de su identidad como mujer, sus capacidades y habilidades para dirigir un grupo o colectivo. Las promotoras Comunales a nivel mundial son mujeres desarrollando su autoconfianza, su autoestima, la conciencia de su identidad y sus derechos; tomando el control de sus vidas; ganando voz y presencia pública a favor de la transformación de las condiciones que mantienen la opresión de género.

El liderazgo femenino es un nuevo paradigma social que permite la emergencia del talento femenino. Según Centeno (2006), los rasgos más distintivos, son entre otros:

- Un estilo más democrático, dialogante, pedagógico, consensuado y mediador. Una actitud más receptiva y participativa.

- Un liderazgo más multidireccional y multidimensional, favoreciendo el desarrollo de valores y acciones colectivas.

- Un ejercicio del liderazgo más firme y constante.

- Más creativas en las propuestas para ejercer la dirección.

- Más comunicativas y abiertas a las relaciones personales, a las propuestas de sus compañeras y compañeros, a escuchar sin enjuiciar de una forma más comprensiva.

- Desarrollan políticas de cooperación y participación.

- Potencian relaciones interpersonales.

- Disponibilidad para el cambio.

- Crean un clima de colegialidad y gestión colaborativa con el profesorado.

- Construyen redes informales, propiciando la cohesión y los vínculos entre los miembros, alentando a otros a compartir los recursos.

- Les preocupa el abuso del poder y utilizan la coacción como último recurso.

- Potencian el "aprender con los demás".

- Clara preferencia por enfoques consultivos y cooperativos.

- Habilidad para combinar y reconciliar expectativas y experiencias contradictorias, promoviendo el compromiso.

- Desarrollo de políticas de cuidado y apoyo mutuo.

Fecha de recepción: 20 de julio, 2010

Fecha de aceptación: 1 de agosto, 2010
- Mayor atención a los sentimientos y al uso de una "inteligencia emocional" más sensible a las emociones y situaciones personales de los demás.

Como corolario a esta presentación hemos querido destacar el rol extraordinario de los millones de mujeres que a nivel mundial hacen posible un desarrollo más humano y solidario y contribuyen al ejercicio de los derechos humanos. En el caso especifico de Perú y Nepal dos países con el poder sus majestuosas montanas, ejemplo del valor de las mujeres especialmente las Promotoras Comunales... Mujeres Descomunales!!!

\section{REFERENCIAS}

Anderson Benedict (1993). Comunidades imaginadas. Una reflexión sobre el origen y la difusión del Nacionalismo. Mexico: Fondo de Cultura Económica.

Brouwer Jani. (1990) Trabajando con Promotores. La Haya: Fundación Bernard Van Leer

Diez G., Javier; Valle, Rosa; Terrón, Eloína; Centeno S., Begoña (2006). El Liderazgo Femenino y su ejercicio en las organizaciones educativas. Revista Iberoamericana de Educación. Disponible en internet: http://www.rieoie.org/deloslectores/ 483Diez.pdf

Freire Paulo. (1964) La educación como práctica de la libertad. Madrid: Siglo XXI.

Freire Paulo. (1970) Pedagogía del Oprimido. Mexico: Siglo XXI.

INFORMET, I. d. (2005). El empoderamiento: significado y alcances en la Lucha contra la pobreza y la exclusion social. En: Empoderamiento un camino para luchar contra la pobreza (p. 14-15). Lima: COSUDE-PERÚ.

Miloslavich Tupac, Diana (1993). María Elena Moyano: En busca de una esperanza. Lima: Centro de la Mujer Peruana Flora Tristán.

Palomino Nancy (1991) Los discursos y la Vida. Reflexiones de un encuentro entre educadores populares y promotores. Lima: Tarea 